\title{
Motion of an Electron from a Point Source in Parallel Electric and Magnetic Fields
}

\author{
Christian Bracher \\ William \& Mary \\ John B. Delos \\ William \& Mary, jbdelos@wm.edu
}

Follow this and additional works at: https://scholarworks.wm.edu/aspubs

Part of the Physics Commons

\section{Recommended Citation}

Bracher, Christian and Delos, John B., Motion of an Electron from a Point Source in Parallel Electric and Magnetic Fields (2006). Physical Review Letters, 96(10).

https://doi.org/10.1103/PhysRevLett.96.100404

This Article is brought to you for free and open access by the Arts and Sciences at W\&M ScholarWorks. It has been accepted for inclusion in Arts \& Sciences Articles by an authorized administrator of W\&M ScholarWorks. For more information, please contact scholarworks@wm.edu. 


\title{
Motion of an Electron from a Point Source in Parallel Electric and Magnetic Fields
}

\author{
Christian Bracher* and John B. Delos \\ Physics Department, The College of William and Mary, Williamsburg, Virginia 23187-8795, USA
}

(Received 5 August 2005; published 15 March 2006)

\begin{abstract}
Negative ions undergoing near-threshold photodetachment in a weak laser field provide an almost pointlike, isotropic source of low-energy electrons. External fields exert forces on the emitted coherent electron wave and direct its motion. Here, we examine the spatial distribution of photodetached electrons in uniform, parallel electric and magnetic fields. The interplay of the electric and magnetic forces leads to a surprising intricate shape of the refracted electron wave, and mutiple interfering trajectories generate complex fringe patterns in the matter wave. The exact quantum solution is best understood in terms of the classical electron motion.
\end{abstract}

DOI: 10.1103/PhysRevLett.96.100404

PACS numbers: 03.75.-b, 32.80.Gc

Wave-particle dualism is one of the strangest and most fascinating aspects of quantum mechanics. In his famous "electron gun" thought experiment [1], Richard Feynman explores what happens if an electron travels simultaneously along two trajectories, and interferes with itself. In the 1980s, Demkov et al. [2] suggested a macroscopic version of Feynman's idea: Photodetachment of negative ions provides a localized source of electrons with fixed energy, and an externally applied homogeneous electric field accelerates them. In a uniform force field, there are always exactly two trajectories that join the source with any given destination in range [3] (a fact already known to Galileo). Along each path, the electron wave function accumulates a phase proportional to the classical action. Therefore, the electron distribution on a detector perpendicular to the electric field displays a set of macroscopic concentric interference fringes. These patterns were recorded experimentally by Blondel et al. with their "photodetachment microscope" [4]. The results are in excellent agreement with theory [5] and in turn allow precision measurements of electron affinities [6]. (Recently, spatially resolved interference structures have also been observed in photoionization microscopy [7].)

These experiments permit observation of matter waves in real space, and they show how to guide an expanding electron cloud using electromagnetic forces. Hence, it is worthwhile to explore photodetachment in more complex field configurations. Here, we predict the photoelectron distribution that will be found on a detector in the presence of parallel, uniform, static electric and magnetic fields $\mathcal{E}=$ $-\mathcal{E} \mathbf{e}_{z}$ and $\mathcal{B}=\mathcal{B} \mathbf{e}_{z}$ [8] that was previously studied only for very weak magnetic fields [9]. (The total photocurrent has been addressed in Ref. [10].)

Near-threshold photodetachment of negative atomic ions provides a virtually pointlike electron source with pure $s$-wave or $p$-wave character, and a sharply defined energy $E$ [11]. (In this Letter we assume $s$-wave emission.) The remaining neutral atomic core interacts very weakly with the ejected electron, and it is an excellent approximation to ignore that interaction entirely, even for closed electron orbits that return to the core; in weak fields, rescattering has been found to be negligible [12].

The dynamics of the electron cloud is thus controlled entirely by the external fields $\mathcal{E}$ and $\mathcal{B}$. Replacing the parent ion with an isotropic point source placed at the coordinate origin, the spreading electron wave obeys the inhomogeneous stationary Schrödinger equation [13]:

$$
[E-\mathcal{H}(\mathbf{r}, \mathbf{p})] \psi(\mathbf{r})=C \delta(\mathbf{r}),
$$

where the details of the photon-ion interaction in the detachment process are summarized in the constant $C$. The Hamiltonian $\mathcal{H}(\mathbf{r}, \mathbf{p})$ for the photoelectron reads

$$
\mathcal{H}(\mathbf{r}, \mathbf{p})=\frac{\mathbf{p}^{2}}{2 m}+\omega_{L}(\mathbf{r} \times \mathbf{p})_{z}+\frac{1}{2} m \omega_{L}^{2} \rho^{2}-e \mathcal{E} z .
$$

Here, $e$ is the elementary charge, $\rho^{2}=x^{2}+y^{2}$ denotes the radial distance, and we introduced the Larmor frequency $\omega_{L}=e \mathcal{B} /(2 m)$.

In order to understand the properties of the resulting electronic wave function $\psi(\mathbf{r})$, it is best to examine the corresponding classical trajectory field. The equations of motion derived from Eq. (2) are easy to solve: Each specific trajectory starts with initial velocity $v_{0}=$ $\sqrt{2 E / m}$ under some polar emission angle $\theta$ measured relative to the field axis, and then undergoes uniform rotation in the polar angle, $\dot{\phi}=\omega_{L}$, harmonic oscillation in the radial coordinate $\rho$ [14], and uniform acceleration in the $z$ direction:

$$
\begin{gathered}
\rho(t)=v_{0} \sin \theta\left|\sin \left(\omega_{L} t\right)\right| / \omega_{L}, \\
z(t)=v_{0} t \cos \theta+e \mathcal{E} t^{2} /(2 m) .
\end{gathered}
$$

The resulting spiraling motion of individual trajectories (blue curves) is shown in Fig. 1 (left panel).

This plot illustrates that the trajectory field possesses much more intricate qualities: The initially radially outgoing trajectories, uniformly distributed over all angles, twist and bend in the external fields, and trace out a complex pattern. The caustics, the envelopes of this family 

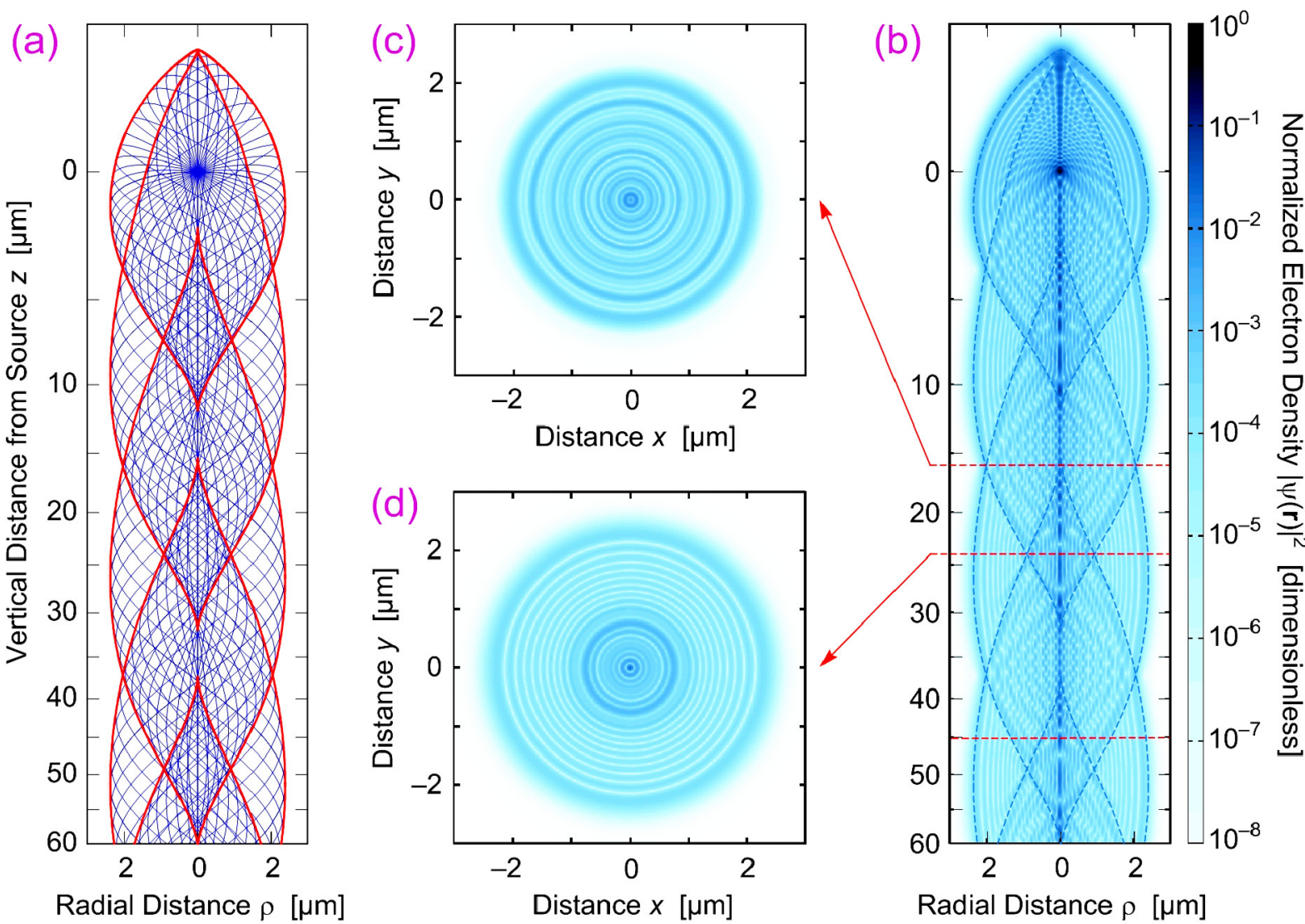

Radial Distance $\rho[\mu \mathrm{m}]$

FIG. 1 (color online). Propagation of $s$-wave photoelectrons, with energy $E=50 \mu \mathrm{eV}$, in parallel electric and magnetic fields $\mathcal{E}=15 \mathrm{~V} / \mathrm{m}, \mathcal{B}=0.02 \mathrm{~T}(\eta=5.592)$. Panels: (a) The classical trajectory field (blue) traces out an infinite series of caustic surfaces in $\rho-z$ space (bold red curves) that begin and end on the symmetry axis. Crossing over a caustic, the number of paths connecting any given point to the source atom at the origin changes by two; sixfold degeneracy occurs in the diamond-shaped areas. (The $z$ axis, here increasing downward, has been scaled to emphasize the periodicity of the pattern.) (b) The exact quantum density distribution features a complex fringe structure that is clearly organized along the classical caustics (overlayed blue dashed curves). Depending on the number of interfering classical trajectories, distinct interference patterns emerge in each region. Because of the focusing property of the magnetic field, the probability to find photoelectrons near the symmetry axis is strongly enhanced. (c) Photodetachment microscope image on a perpendicular detector plane, placed near a constriction of the pattern $(z=16.06 \mu \mathrm{m})$. Four-path interference leads to a complex pattern with evident "superstructure." (d) Photodetachment microscope image at a distance $z=24.3 \mu \mathrm{m}$. Simple, regular two-path interference dominates the outer areas of the image. A double caustic (dark fringe) separates them from the inner region, where sixfold degeneracy prevails. In the center of both images, excess density is observed near the "focal line." A radial density profile of the electron distribution at a distance $z=45 \mu \mathrm{m}$ (red dotted line) is displayed in Fig. 3.

of paths, form an infinite sequence of closed, overlapping, cylindrically symmetric surfaces (bold red curves) that usually begin and end on the symmetry axis in cusp singularities $[10(\mathrm{c}), 10(\mathrm{~d})]$. The geometrical shape of the set of caustics is determined by a single parameter, the ratio $\eta$ of the magnetic and electric forces acting on the electron:

$$
\eta=v_{0} \mathcal{B} / \mathcal{E}
$$

The caustic surfaces are associated with the periodic cyclotron motion: The trajectory field carves out the $k$ th caustic in the interval $(k-1) T_{c} \leq t \leq k T_{c}$, where $T_{c}=$ $\pi / \omega_{L}$. The cusps are related to trajectories emitted parallel to the fields $(\theta=0, \pi)$ and complete cyclotron orbits $(t=$ $\left.k T_{c}\right)$. Maximum radial extension $\left(\rho_{\max }=v_{0} / \omega_{L}\right)$ occurs for perpendicular emission $\left(\theta=\frac{\pi}{2}\right)$ and half-complete or- bits $\left[t=\left(k-\frac{1}{2}\right) T_{c}\right]$. Because of the acceleration in the electric field, the caustics stretch more and more in the $z$ direction as $k$ grows; at large $z$, their pattern becomes periodic in $\sqrt{z}$ (see Fig. 1). A detailed description of the structure of the caustics, and their evolution with changing $\eta$ is given in a separate paper [15].

Caustics are boundaries between classically allowed and forbidden motion: Each time we pass a caustic in an outward direction, a pair of trajectories joining the source with the destination point $\mathbf{r}$ ceases to exist. For fixed $\mathbf{r}$, the number of interfering classical paths is twice the number of caustics enclosing it. Allowing $\mathbf{r}$ to vary $(\rho>0)$, we find for the maximum count of interfering orbits:

$$
N_{\max }=2\lfloor\eta / \pi\rfloor+4,
$$


where $\lfloor\ldots\rfloor$ denotes the integer part. (In Fig. 1, $\eta \sim 5.59$, and maximum degeneracy is sixfold.) Thus, the system allows us to adjust the number of classical trajectories simply by changing $E$ or the field strengths $\mathcal{E}, \mathcal{B}$. We note that fourfold degeneracy occurs even at the weakest magnetic fields (Fig. 2) [9].

Points with $\rho=0$ are exceptional because an infinite number of trajectories (with fixed $\theta$, but arbitrary azimuthal emission angle) may intersect there after a full cyclotron orbit. They generate "focal line segments" on the symmetry axis that connect the cusp singularities.

Using these classical paths, we construct a semiclassical approximation to the wave function [16]:

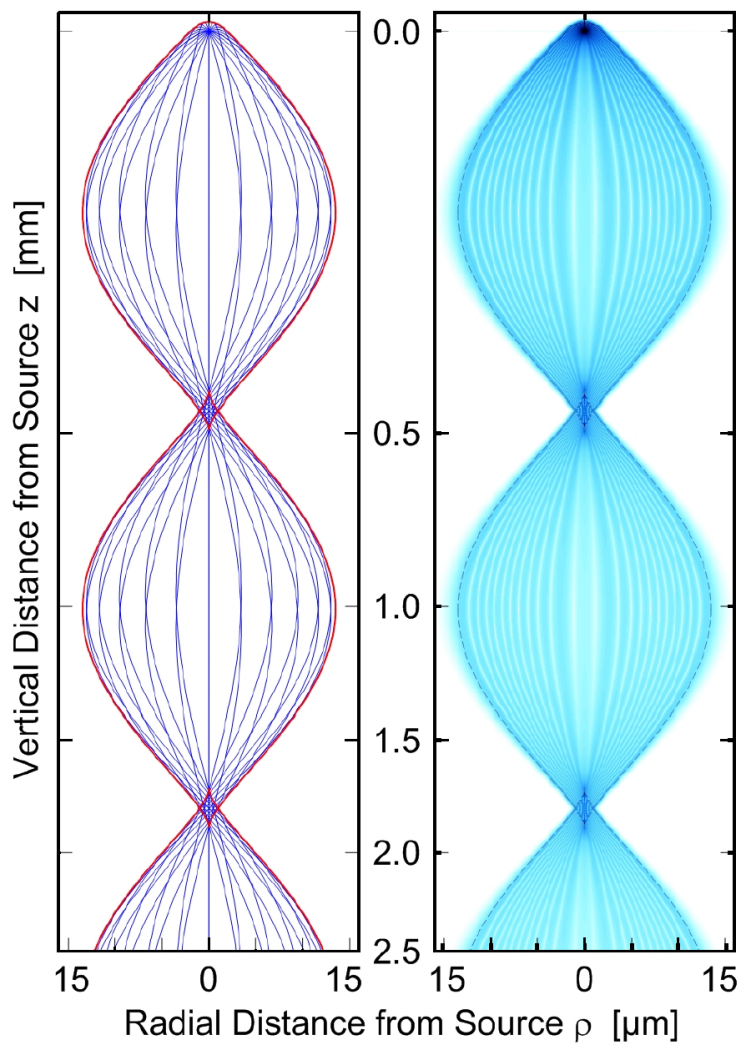

FIG. 2 (color online). Distribution of photodetached electrons (energy $E=100 \mu \mathrm{eV}$ ) in parallel fields $\mathcal{E}=100 \mathrm{~V} / \mathrm{m}$ and $\mathcal{B}=$ $5 \mathrm{mT}(\eta=0.297)$. Left panel: Classical trajectory field (blue curves) and caustics (bold red curves). The pattern expands and contracts in the rhythm of the cyclotron motion. In the small diamond-shaped focal regions, centered around $z_{k}=$ $e \mathcal{E} k^{2} T_{c}^{2} /(2 m)$, the electrons return to the symmetry axis, and four paths link each point to the source. Between the foci, the degeneracy is twofold. - The exact density distribution (right panel) is delineated by the caustics. The focal regions present a series of "bottlenecks" for the photocurrent characterized by high electron density $|\psi(\mathbf{r})|^{2}$ and a complex interference pattern. Outside the foci, the regular two-path interference pattern prevails, and the charge distribution resembles the purely electric case $[2,6]$.

$$
\psi_{\mathrm{sc}}(\mathbf{r})=\sum_{\nu=1}^{N(\mathbf{r})} \sqrt{\rho_{\nu}(\mathbf{r})} \exp \left[\frac{i}{\hbar} \mathcal{W}_{\nu}(\mathbf{r})-\frac{i \pi}{2} \mu_{\nu}(\mathbf{r})\right]
$$

Here, $\rho_{\nu}(\mathbf{r})$ is the classical density along the $\nu$ th trajectory at the endpoint $\mathbf{r}, \mathcal{W}_{\nu}(\mathbf{r})$ is the corresponding classical action, and the Maslov index $\mu_{\nu}(\mathbf{r})$ counts how often the trajectory has touched a caustic surface, or crossed through a focal line, before arriving at $\mathbf{r}$. For improved accuracy of the approximation, we also include the exponentially suppressed contributions of tunneling trajectories [3]. Figure 3 indicates that Eq. (7) yields an excellent approximation to the exact density profile, except at the caustics, where it diverges. An Airy uniform approximation based on the classical paths corrects this failure, and delivers results virtually indistinguishable from the quantum calculation [17].

A section of the exact electron density $|\psi(\mathbf{r})|^{2}$ is shown in panel (b) to the right in Fig. 1. To calculate it, we note that the wave function is just a multiple of the energy Green function $G(\mathbf{r} ; E)[18]$ :

$$
\psi(\mathbf{r})=C G(\mathbf{r} ; E),
$$

that fulfils outgoing-wave boundary conditions. The Hamiltonian (2) is separable, $\mathcal{H}=\mathcal{H}_{\perp}+\mathcal{H}_{\|}$, so the energy $E_{\perp}$ bound in the radial motion, and the energy $E_{\|}=$ $E-E_{\perp}$ available for motion in $z$ direction, are conserved individually. The spectrum $\left\{E_{\perp}\right\}$ of the simple harmonic oscillator $\mathcal{H}_{\perp}$ is discrete; its eigenstates $\chi_{n}(\rho)$ occupy the Landau levels $E_{n}=(2 n+1) \hbar \omega_{L}$ [19]. Propagation takes place only in the field direction where the electrons undergo uniform acceleration, as described by the onedimensional Green function $G_{\|}\left(z ; E_{\|}\right)$for the Hamiltonian $\mathcal{H}_{\|}$[3]. We then expand the full Green function $G(\mathbf{r} ; E)$ into a series over the product states, the scattering channels $\chi_{n}(\rho) G_{\|}\left(z ; E-E_{n}\right)$ :

$$
\begin{aligned}
G(\mathbf{r} ; E)= & -\frac{m \mathcal{B}}{\beta \hbar^{3} \mathcal{E}} \exp \left(-\frac{m \omega_{L}^{2} \rho^{2}}{2 \hbar}\right) \\
& \times \sum_{n=0}^{\infty} L_{n}\left(\frac{m \omega_{L}^{2} \rho^{2}}{\hbar}\right) \operatorname{Ai}\left[-\beta\left(E-E_{n}\right)\right] \\
& \times \operatorname{Ci}\left[-\beta\left(E-E_{n}+e \mathcal{E} z\right)\right] .
\end{aligned}
$$

The inverse energy $\beta$ is defined via $\beta^{3}=2 m /(\hbar e \mathcal{E})^{2}$, and $L_{n}(u)$ denotes a Laguerre polynomial. $\operatorname{Ai}(u)$ and $\operatorname{Ci}(u)=$ $\mathrm{Bi}(u)+i \mathrm{Ai}(u)$ are, respectively, the regular and outgoingwave Airy functions. The result holds for $z>0$; for $z<0$, the arguments of the Airy functions must be exchanged. Except in the limit $z \rightarrow 0$, the series (9) converges rapidly.

Conceptually, the semiclassical sum over trajectories (7) and the quantum mechanical series involving Landau levels (9) are starkly different. Yet, Fig. 1 reveals at once that the classical trajectory field is the key to an understanding of the exact solution: The caustics clearly delineate the features of the quantum distribution, and the regions with 


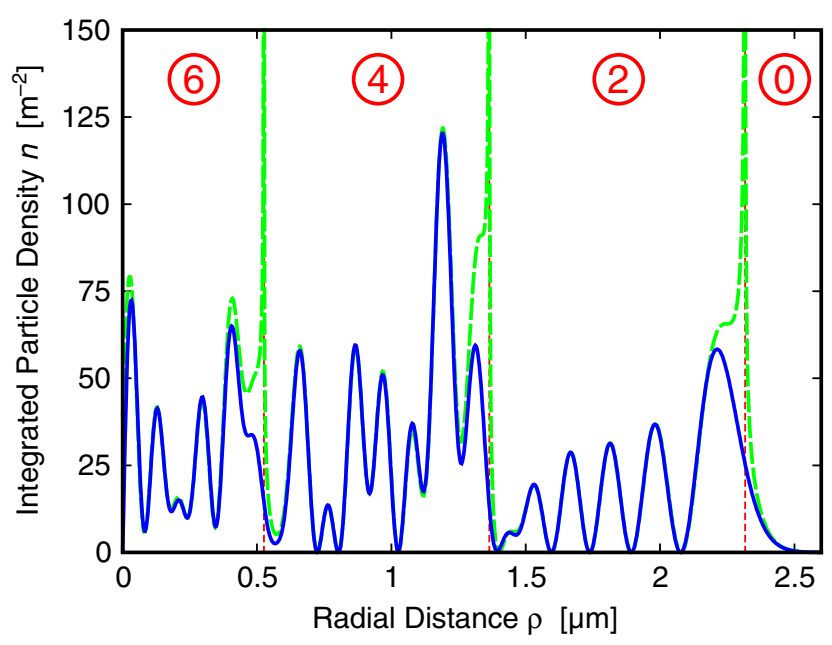

FIG. 3 (color online). Integrated density profile $n(\rho)=$ $2 \pi \rho|\psi(\rho)|^{2}$ of the detached photoelectrons, for normalized flux $1 \mathrm{~s}^{-1}$, at a distance $z=45 \mu \mathrm{m}$ from the source. (Parameters as in Fig. 1.) Blue (solid) curve: Exact quantum result; green (dashed) curve: Semiclassical approximation, including tunneling trajectories. This approximation diverges at the caustics (red dotted lines), where the number of classical trajectories (circled numbers) connecting the atom and the destination point changes. The divergences can be corrected using uniform approximations.

two, four, or six interfering paths each possess their own distinctive fringe structures. Two sections of the electron density, i.e., photodetachment microscope images in parallel fields, are depicted in panels (c) and (d) (center column in Fig. 1). Close to the caustics, and similarly near the focal line at the center of the image, the density is particularly high (dark rings). Regular oscillations reminiscent of Blondel's observations [4,6] emerge in the outer region where only two paths interfere, whereas at smaller $\rho$, the presence of four or more classical trajectories leads to more involved patterns.

To summarize, near-threshold photodetachment provides an almost perfect coherent point source of free electrons. External fields may be used to guide them and exert "quantum control" over the propagating wave: For motion in parallel homogeneous fields, the number of interfering classical paths can be adjusted simply by tuning the parameter $\eta(5)$. Furthermore, this analytically solvable problem serves to illustrate the power of the semiclassical method.

A practical problem remains: For typical field strengths used in photodetachment microscopy experiments [6], the predicted patterns (while large on an atomic scale) might be too small to be resolved. In a future Letter we will consider detachment in parallel, nonuniform field configurations that "flare out" and magnify the interference pattern on the detector.
We appreciate valuable discussions with T. Kramer. This work was supported by NSF.

*Electronic address: cbracher@brynmawr.edu

[1] R. P. Feynman, R. B. Leighton, and M. Sands, The Feynman Lectures on Physics (Addison-Wesley, Reading, MA, 1964), Vol. III.

[2] Y. N. Demkov, V. D. Kondratovich, and V. N. Ostrovskii, JETP Lett. 34, 403 (1982) [Pis'ma Zh. Eksp. Teor. Fiz. 34, 425 (1981)].

[3] C. Bracher, W. Becker, S. A. Gurvitz, M. Kleber, and M. S. Marinov, Am. J. Phys. 66, 38 (1998).

[4] C. Blondel, C. Delsart, and F. Dulieu, Phys. Rev. Lett. 77, 3755 (1996)

[5] V. Z. Slonim and F. I. Dalidchik, Sov. Phys. JETP 44, 1081 (1976) [Zh. Eksp. Teor. Fiz. 71, 2057 (1976)].

[6] F. Goldfarb, C. Drag, W. Chaibi, S. Kröger, C. Blondel, and C. Delsart, J. Chem. Phys. 122, 014308 (2005); C. Blondel, W. Chaibi, C. Delsart, C. Drag, F. Goldfarb, and S. Kröger, Eur. Phys. J. D 33, 335 (2005).

[7] C. Nicole, H.L. Offerhaus, M.J.J. Vrakking, F. Lépine, and C. Bordas, Phys. Rev. Lett. 88, 133001 (2002); F. Lepine, C. Bordas, C. Nicole, and M. J. J. Vrakking, Phys. Rev. A 70, 033417 (2004).

[8] For some results regarding electron propagation in a uniform, purely magnetic field, see: M. V. Berry, Eur. Phys. J. 2, 22 (1981).

[9] T. Kramer, C. Bracher, and M. Kleber, Europhys. Lett. 56, 471 (2001).

[10] (a) M.L. Du, Phys. Rev. A 40, 1330 (1989); (b) I. I. Fabrikant, Phys. Rev. A 43, 258 (1991); (c) A. D. Peters, C. Jaffé, and J. B. Delos, Phys. Rev. Lett. 73, 2825 (1994); (d) Phys. Rev. A 56, 331 (1997); (e) Q. Wang and A. F. Starace, Phys. Rev. A 51, 1260 (1995).

[11] In this Letter, we ignore effects of the electron spin.

[12] I. I. Fabrikant, J. Phys. B 27, 4545 (1994); N. D. Gibson, M. D. Gasda, K. A. Moore, D. A Zawistowski, and C. W. Walter, Phys. Rev. A 64, 061403(R) (2001).

[13] L.S. Rodberg and R.M. Thaler, Introduction to the Quantum Theory of Scattering (Academic, New York, 1967); T. Kramer, C. Bracher, and M. Kleber, J. Phys. A 35, 8361 (2002).

[14] In other words, cyclotron motion with frequency $\omega_{C}=$ $2 \omega_{L}$ takes place in the $x-y$ plane. One may imagine that the trajectories in Fig. 1 are drawn in a frame precessing at the Larmor frequency.

[15] C. Bracher, T. Kramer, and J. B. Delos (to be published).

[16] J. B. Delos, Adv. Chem. Phys. 65, 161 (1986).

[17] The approximation may still fail on the symmetry axis. There, focal lines, cusps, and umbilic points require increasingly complex uniform approximation schemes. See also A. D. Peters, C. Jaffé, J. Gao, and J. B. Delos, Phys. Rev. A 56, 345 (1997).

[18] E. N. Economou, Green's Functions in Quantum Physics (Solid-State Sciences 7) (Springer, Berlin, 1983).

[19] B. R. Johnson, J. O. Hirschfelder, and K. H. Yang, Rev. Mod. Phys. 55, 109 (1983). 